\title{
ECTOPARASITE BURDENS OF THE COMMON MOLE-RAT (CRYPTOMYS HOTTENTOTUS HOTTENTOTUS) FROM THE CAPE PROVINCES OF SOUTH AFRICA
}

\author{
Elizabeth K. Archer, Nigel C. Bennett, Edward A. Ueckermann*, and Heike Lutermann \\ Mammal Research Institute, Department of Zoology and Entomology, University of Pretoria, Pretoria 0002, South Africa. Correspondence should be sent to: \\ hlutermann@zoology.up.ac.za
}

\begin{abstract}
The members of the African mole-rat family Bathyergidae are widely distributed across sub-Saharan Africa. Despite their well-studied biology and reproductive physiology, the current knowledge of their ectoparasite fauna is limited and ambiguous due to recent revisions of the bathyergid taxonomy. The common mole-rat (Cryptomys hottentotus hottentotus) is 1 of the most widely distributed species of these subterranean rodents. Ectoparasites were collected from 268 common mole-rats at 2 localities (Western and Northern Cape provinces) in South Africa over the course of 18 mo with the aim to document species richness, prevalence, and abundance of these ectoparasites. The aggregation of parasite species, sex bias within a species, and seasonal variation in ectoparasite burdens were investigated. A total of 4,830 individual parasites from 4 mite species (Androlaelaps scapularis, Androlaelaps capensis, Radfordia ensifera, and 1 undetermined chigger [family Trombiculidae]), 1 flea species (Cryptopsylla ingrami), and 1 louse species (Eulinognathus hilli) were collected. With the exception of $R$. ensifera and the chigger, all of these ectoparasites appear to be host specific either for the host species or the Bathyergidae. Aggregation indices indicated that with the exception of $E$. hilli, the distribution of all parasite species was highly aggregated among hosts and sex biased. Seasonal variation in prevalence, abundance, and species richness was apparent, with greater burdens in the rainy winter season. This is likely related to seasonal variation in abiotic factors but may also be affected by the timing of host reproduction and dispersal behavior.
\end{abstract}

The distribution of a parasite community across a population of hosts is influenced by many factors and tends to be aggregated, with only a few host individuals carrying the majority of the parasites (Wilson et al., 2001; Poulin, 2007). This heterogeneity can be affected by various biotic and abiotic factors, such as temperature, rainfall, and humidity (Schalk and Forbes, 1997; Wilson et al., 2001; Moore and Wilson, 2002; Klein, 2004). Seasonal variation in parasite burdens is a particularly common pattern in both endo- and ectoparasites (Wilson et al., 2001; Altizer et al., 2006). However, the influences of the environment on parasite populations tend to be more pronounced in ectoparasites such as fleas and mesostigmatid mites (Krasnov, 2008; Korallo-Vinarskaya et al., 2009). Ectoparasites that have developmental stages that live off the host are thought to be particularly susceptible to effects associated with the surrounding habitat (Vinarski et al., 2007; Krasnov, 2008). However, no taxon-specific pattern is apparent, and although some studies have shown that lower levels of humidity can reduce the abundances (Krasnov, 2008; Viljoen et al., 2011), others have found the opposite pattern (Fabiyi, 1996; Matthee et al., 2007).

Seasonal changes in parasite abundances are not always thought to be a direct result of abiotic fluctuations and might affect abundance indirectly through their effects on hosts (Altizer et al., 2006; Martin et al., 2008). Host breeding season can lead to increased population densities that enhance potential for horizontal transmission of parasites. Furthermore, changes in hormonal balances of the host during the reproductive period can alter immune function and therefore susceptibility to parasitic infestation (Christe et al., 2000; Klein, 2004). Because the breeding season for many host species coincides with times of higher resource availability, some ectoparasites

Received 8 April 2013; revised 9 October 2013; accepted 14 October 2013.

* ARC-Plant Protection Research Institute, Private Bag X134, Queenswood, Pretoria, 0121, South Africa; and School of Environmental Sciences and Development, Potchefstroom University, Potchefstroom, 2520, South Africa.

DOI: $10.1645 / 13-270.1$ also synchronize their reproduction with that of their hosts (Rothschild and Ford, 1964; Christe et al., 2000; Lourenço and Palmeirim, 2008).

African mole-rats (family Bathyergidae) are subterranean hystricomorph rodents widely distributed throughout subSaharan Africa (Bennett and Faulkes, 2000). Although well studied with regard to their social system and reproductive physiology, studies investigating their parasite fauna remain scarce, and only a limited number of hosts have been sampled (Scharff et al., 1997; Tenora et al., 2003; Viljoen et al., 2011). This is particularly true for their ectoparasite fauna, and several parasitological studies of bathyergid species state explicitly that they did not find any ectoparasites (De Graaff, 1972; Scharff et al., 1997). In contrast, older records indicate a diverse array of ectoparasites, including mites, fleas, lice, and 1 tick species that have been collected from members of this family (reviewed in De Graaff, 1981). However, the host identity of many of these records is questionable due to the lack of locational information and the extensive revisions that the Bathyergidae have undergone over recent years (Faulkes et al., 2004; Ingram et al., 2004; Van Daele et al., 2007). As a consequence, only the 4 solitary members of the family and the naked mole-rat (Heterocephalus glaber) retained their species names; the remaining species have been split into 2 genera. Furthermore, the former species Cryptomys hottentotus has been divided into several subspecies that might be sufficiently distinct to merit species level classifications (Faulkes et al., 1997; Ingram et al., 2004). The common mole-rat (Cryptomys hottentotus hottentotus) is the most widespread of the latter group, ranging from the Cape Peninsula northward to Steinkopf near the Namibian border and inland through the eastern Cape, Natal, and the Western and Northern Transvaal (Bennett and Faulkes, 2000).

In the current study, we extensively assessed the ectoparasite burdens of the common mole-rat to (1) give an updated account of the ectoparasite fauna sustained by this species. Furthermore, (2) we investigated the aggregation and possible sex biases among adult ectoparasites. Lastly, (3) we evaluated the effect of season on ectoparasite burdens. 
TABLE I. Arthropod ectoparasites found on Cryptomys hottentotus hottentotus sampled between February 2011 and August 2012 in the Northern and Western Cape provinces, South Africa.

\begin{tabular}{|c|c|c|c|c|}
\hline Taxon & Order & Suborder/superfamily & Family & Species \\
\hline & & Prostigmata & Myobiidae & Radfordia ensifera (Poppe, 1896) \\
\hline Fleas & Siphonaptera & Hystrichopsylloidea & Chimaeropsyllidae & Cryptopsylla ingrami (DeMeillon, 1938) \\
\hline Lice & Phthiraptera & Anoplura & Polyplacidae & Eulinognathus hilli (Bedford, 1929) \\
\hline
\end{tabular}

\section{MATERIALS AND METHODS}

Common mole-rats were captured from 2 sites representing the southern and northern edges of the distributional range. The first site, Waylands Farm, was situated just outside of Darling, Western Cape $\left(33.25^{\circ} \mathrm{S}, 18.25^{\circ} \mathrm{E}\right)$. The second site, Pedroskloof Farm, was located $25 \mathrm{~km}$ outside of Kamieskroon, Northern Cape, in the Kamiesberg Mountains $\left(30.13^{\circ} \mathrm{S}, 17.57^{\circ} \mathrm{E}\right)$ approximately $440 \mathrm{~km}$ north of Darling. Capture trips lasted between 2 and $3 \mathrm{wk}$ and were conducted during both the austral summer (January-March) and winter (June-August) from February 2011 to August 2012. Four capture trips lasting between 2 and 3 wk each were in Darling, whereas only 3 trips could be carried out in Kamieskroon. Animals were captured with Hickman live traps (Hickman, 1979) that were baited with sweet potato and placed in exposed tunnels of a burrow system. Traps were checked every $2-3 \mathrm{hr}$ from dusk to dawn until activity at a capture site had ceased for more than $48 \mathrm{hr}$. Any animals caught were kept on a diet of sweet potato in plastic crates with at least a 3-cm layer of soil until processed.

Individual hosts were killed with an overdose of halothane within a week of capture, and a modified washing technique was applied (Henry and McKeever, 1971). Each individual was washed thoroughly in $100 \mathrm{ml}$ of soapy water in a $22 \times 7 \times 5$-cm tub to remove any ectoparasites. The washing process was standardized for all individuals and involved kneading through the fur with the fingertips all the way along and around the body length 5 times, before washing the head and the posterior end. Then, individuals were moved back and forth through the water, first on their front and then on their back (10 times each side), to remove any ectoparasites that remained caught in the fur. This wash-water was transferred to a labeled screw-top container until processed. Each wash sample was filtered through a no. 25 U.S. Standard Sieve (710- $\mu \mathrm{m}$ screen) for ectoparasites and screened for ectoparasites under a binocular dissection microscope. Any ectoparasites found were placed into Eppendorf tubes containing $70 \%$ ethanol. Specimens were prepared for identification following standard protocols and identified to species with the use of identifications handbooks and reference keys (Till, 1963; Ledger, 1980; Segerman, 1995; Baker, 1999). Representative samples of the ectoparasites were deposited with the South African Agricultural Research Council in Pretoria, South Africa, as accession numbers Acy 13/ 499-13/505. Following the definitions provided by Bush et al. (1997), we calculated the proportion of hosts in populations infested (prevalence) and mean number of parasites on a host (abundance) of each parasite species using the program Quantitative Parasitology 3.0 (Rózsa et al., 2000). We calculated the dispersion index $(D)$ as a measure of aggregation for each parasite species (Poulin, 1993) using the program Quantitative Parasitol- ogy 3.0 (Rósza et al., 2000). The dispersion index ranges from 0 to 1 and greater values indicate increasing degrees of aggregation among a parasite population. In addition, we calculated the number of parasite species per host (species richness). To test for the effects of season on parasite prevalence and adult sex ratios, we used chi-square tests. Mann-Whitney $U$-tests were used to compare abundance and species richness between seasons.

\section{RESULTS}

Ectoparasites were collected from 268 common mole-rat individuals (summer: $\mathrm{n}=74$; winter: $\mathrm{n}=194$ ). In total, we collected 4,830 individual parasites from these mole-rats, resulting in an overall ectoparasite prevalence of $78.4 \%$ (210/268 individuals). These ectoparasites were from 6 species: 4 mite, 1 flea, and 1 sucking louse species (Table I). No ticks were found on any individual. The majority of host animals were infested with 1 or 2 ectoparasite species (26.9 and $27.6 \%$, respectively), and $18.3 \%$ harbored 3 species. Only $4.9 \%$ carried 4 ectoparasite species, and $0.7 \%$ was infested with 5 species. As well as being the most speciose, mites were the most abundant parasites (Table II). The mesostigmatic mite Androlaelaps scapularis alone comprised $78.3 \%$ of all the parasites collected. In contrast, the other member of this genus, Androlaelaps capensis, was much less prevalent and abundant (Table II). With 1 exception, animals harboring this species were always co-infected with $A$. scapularis. The second most prevalent ectoparasite species was the prostigmatic mite Radfordia ensifera; however, it only occurred at a low abundance (Table II). A single chigger specimen was found on $C$. $h$. hottentotus. Of the remaining ectoparasite species, the flea Cryptopsylla ingrami was most prevalent (Table II). The louse Eulinognathus hilli was only collected from Kamieskroon, and $98.6 \%$ of these were collected during 1 particular winter trip. Regardless, this was the second most abundant ectoparasite species (Table II).

The index of discrepancy indicated that all of the parasite species had an aggregated distribution (Table II). The degree of

TABLE II. Population structure of ectoparasite species harbored by Cryptomys hottentotus hottentotus sampled from the Northern and Western Cape provinces of South Africa between February 2011 and August 2012.

\begin{tabular}{lccc}
\hline \multicolumn{1}{c}{ Species } & \% Prevalence $(95 \%$ CI*) & Abundance $(95 \% \mathrm{CI})$ & $D$ \\
\hline Androlaelaps scapularis & $73.1(64.7-78.2)$ & $14.05(11.28-17.07)$ & $0.72(0.45-1.02)$ \\
Androlaelaps capensis & $21.6(16.9-27.0)$ & $1.29(1.05-1.57)$ & 0.707 \\
Radfordia ensifera & $43.3(34.5-46.3)$ & $0.28(0.20-0.38)$ & 0.766 \\
Cryptopsylla ingrami & $16.0(12.1-21.0)$ & $1.59(0.88-2.41)$ & 0.866 \\
Eulinognathus hilli & $8.2(5.4-12.1)$ & 0.951 \\
\hline
\end{tabular}

* CI, confidence interval. 
TABLE III. Distribution of developmental stages and sexes of the ectoparasite species of Cryptomys hottentotus hottentotus sampled in the Northern and Western Cape provinces of South Africa between February 2011 and August 2012.

\begin{tabular}{lrccrrrrr}
\hline \multicolumn{1}{c}{ Species } & Total & Male (\%) & Female (\%) & Sex ratio (o: : $)$ & $\chi^{2}$ & $P$ & Nymph (\%) & Larva (\%) \\
\hline Androlaelaps scapularis & 3,785 & 6.58 & 54.77 & $1: 8.33$ & 1432.81 & $<0.001$ & 37.89 & 0.71 \\
Androlaelaps capensis & 194 & 54.12 & 37.11 & $1: 0.69$ & 6.15 & 0.016 & 7.73 & $-*$ \\
Radfordia ensifera & 347 & 55.91 & 32.85 & $1: 0.59$ & 20.78 & $<0.001$ & 7.49 & 2.59 \\
Cryptopsylla ingrami & 76 & 64.47 & 32.89 & $1: 0.51$ & 7.78 & 0.007 & - & - \\
Eulinognathus hilli & 427 & 17.10 & 21.08 & $1: 1.23$ & 1.77 & 0.210 & 58.08 & - \\
\hline
\end{tabular}

*-, stage not present.

aggregation decreased with increasing prevalence of a species. The majority of $A$. scapularis collected were adults, and the sex ratio among those adults was significantly biased toward females (Table III). Immature stages were rarer for the other 2 common mite species, $A$. capensis and $R$. ensifera. Although they exhibited a significant sex bias, the majority of specimens were male (Table III). Similarly, C. ingrami exhibited a significant male bias (Table III). Unlike in the other ectoparasite species, the majority of lice were nymphs, and no significant bias toward either sex was observed among adults.

All of the ectoparasite species found were significantly more prevalent and abundant in winter (Figs. 1, 2; Table IV). In fact, $C$. ingrami was only found in winter, and $A$. capensis and $E$. hilli were almost exclusive to winter field trips. Due to the larger abundances of parasites in winter, host animals exhibit a greater parasite species richness during winter compared with summer (Mann-Whitney test, $U=8.41, P<0.001$; Fig. 3).

\section{DISCUSSION}

Overall, we found a limited number of ectoparasite species on C. h. hottentotus. Most of these species have been collected in previous studies on mole-rats referred to as C. hottentotus (De Graaff, 1964, 1981). The low number of species found does appear to be a typical pattern for mole-rats based on previous studies that have investigated the ectoparasites of bathyergids (De Graaff, 1964, 1981; Viljoen et al., 2011). This impoverished endoparasite fauna may be a result of limited parasite exposure in the subterranean habitat of such rodents (Rossin et al., 2010; Viljoen et al., 2011). We cannot exclude the possibility that our sampling approach might have missed additional ectoparasites

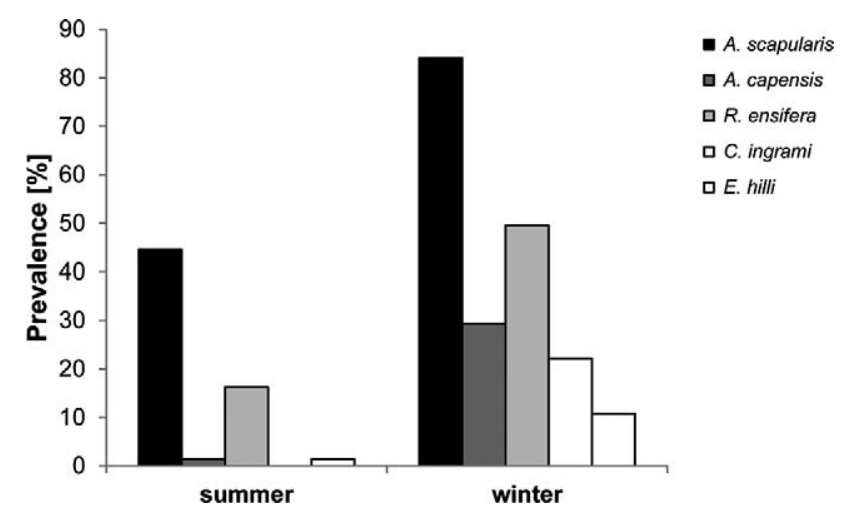

Figure 1. Seasonal variation in ectoparasite prevalence of Cryptomys hottentotus hottentotus sampled in the Northern and Western Cape provinces of South Africa between February 2011 and August 2012. and that the lack of ticks encountered in this study is partially attributable to animals being sampled several days after capture. However, De Graaff (1964) points out that only few ticks have ever been found on mole-rats; and for the only tick species that has ever been found on the study species, Ixodes alluaudi, Neumann 1913, common mole-rats are most likely accidental hosts. Furthermore, the large host sample size and seasonal sampling should ensure that the results produced from this study provide a good representation of ectoparasite infestations in this species.

As in a recent study in a closely related species (Viljoen et al., 2011), the highveld mole-rat (Cryptomys hottentotus pretoriae), mites were the most speciose ectoparasites found on $C . h$. hottentotus. Furthermore, as in the current study, the ectoparasite assemblage of highveld mole-rats was dominated by $A$. scapularis both in terms of prevalence and abundance. Viljoen et al. (2011) also reported $A$. capensis from highveld mole-rats at lower prevalence and abundance as observed in the current study. Both of these mite species have only been recorded from bathyergids (Zumpt, 1961; De Graaff, 1981; Viljoen et al., 2011), suggesting host specificity at the family level. Androlaelaps scapularis has been recorded from the Cape dune mole-rat (Bathyergus suillus), and $A$. capensis has been found on the Cape mole-rat (Georychus capensis) (Zumpt, 1961; De Graaff, 1981). Both of these bathyergids occur sympatrically, with the common mole-rat in the southern part of its distributional range, including our southern sampling site (Bennett and Faulkes, 2000). Because $A$. cryptomius was absent in the present study, this might indicate that it is host specific at the species level and hence would not parasitize $C$. h. hottentotus. The Namaqua dune mole-rat (Bathyergus janetta) occurs sympatrically with the study species

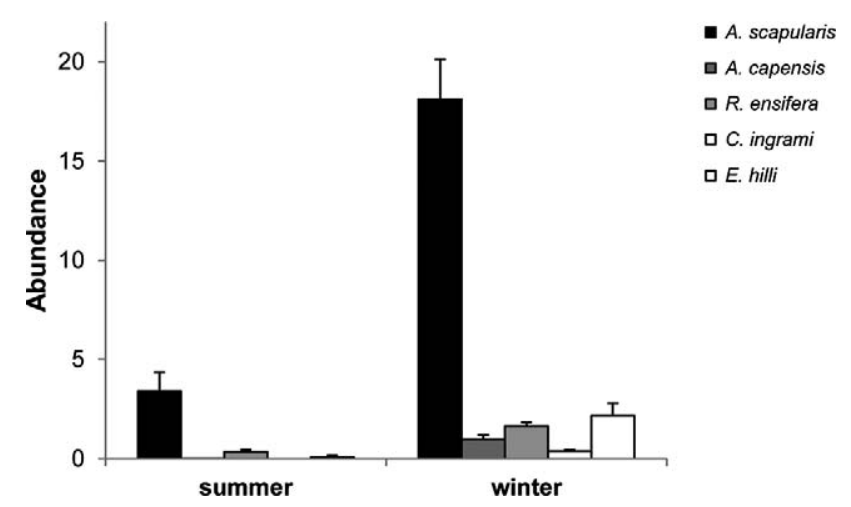

FIGURE 2. Seasonal variation in ectoparasite abundance of Cryptomys hottentotus hottentotus sampled between February 2011 and August 2012 in the Northern and Western Cape provinces, South Africa. 
TABLE IV. Results for the comparison between winter and summer of prevalence and abundance of ectoparasites sampled in the Northern and Western Cape provinces of South Africa between February 2011 and August 2012

\begin{tabular}{lrrrrr}
\hline & \multicolumn{2}{c}{ Prevalence } & & \multicolumn{2}{c}{ Abundance } \\
\cline { 2 - 3 } \cline { 5 - 6 } \multicolumn{1}{c}{ Species } & \multicolumn{1}{c}{$\chi^{2}$} & $P$ & & $U$ & \multicolumn{1}{c}{$P$} \\
\hline Androlaelaps scapularis & 42.38 & $<0.001$ & & 7.00 & $<0.001$ \\
Androlaelaps capensis & 24.82 & $<0.001$ & & 4.97 & $<0.001$ \\
Radfordia ensifera & 24.64 & $<0.001$ & & 5.06 & $<0.001$ \\
Cryptopsylla ingrami & 19.54 & $<0.001$ & & 4.39 & $<0.001$ \\
Eulinognathus hilli & 6.83 & 0.012 & & 2.54 & 0.011 \\
\hline
\end{tabular}

in the northern sampling location; however, because its ectoparasite burden has never been assessed, no inferences can be made.

Radfordia ensifera has not previously been reported from any member of the Bathyergidae, although another member of the family Myobiidae has been recorded (Zumpt, 1961). That this is the first record of $R$. ensifera for a bathyergid species may be attributable to the small number of individuals sampled for this host family and to the sampling in an agricultural habitat, because such transformed habitats are often inhabited by high densities of murid rodents that commonly host this mite. This species, like other members of the family, appears to be a generalist ectoparasite with a global distribution (Fain et al., 1980; Baker, 1999; Soliman et al., 2001). However, although it is prevalent in the host population, its abundance was low.

The remaining ectoparasite species seem to be specialists because both $C$. ingrami and $E$. hilli have previously been recorded only from C. hottentotus (Zumpt, 1966; Ledger, 1980; Durden, 1991; Segerman, 1995; this study). Cryptopsylla ingrami is the only member of the Chimaeropsyllidae that lacks eyes, possibly as an adaptation to living in the sealed burrow systems of its host (Segerman, 1995). The current study represents its northernmost record to date (Segerman, 1995). We collected $E$. hilli from Kamieskroon but not from Darling, whereas it has previously been recorded in Natal Province and Botswana (Ledger, 1980; Durden, 1991). The closely related louse Eulinognathus lawrensis (Bedford, 1929) has been collected from B. suillus and is also thought to be host specific (Ledger, 1980).

All of the ectoparasite species infesting common mole-rats were highly aggregated, with a few individuals harboring the majority of parasites, as is frequently observed for parasite populations (Wilson et al., 2001; Poulin, 2007). Such aggregation could have implications for the sex ratio of ectoparasite species due to the isolation of infrapopulations on individual hosts (Marshall, 1981; Poulin, 2007). The increasing isolation of such infrapopulations with decreasing ectoparasite prevalence and a resulting increased risk of inbreeding has been invoked to explain the commonly observed female bias of ectoparasites (Marshall, 1981; Matthee et al., 2007; Krasnov, 2008). In such populations, only a small number of males are required to fertilize all females, and females exhibit female-biased offspring sex ratios, resulting in reduced local mate competition (Hamilton, 1967). However, a marked female bias was only observed in the most prevalent ectoparasite species in the current study. Alternatively, sex biases in ectoparasite species have been explained with sex differences in mobility, longevity, or susceptibility to abiotic condition or host

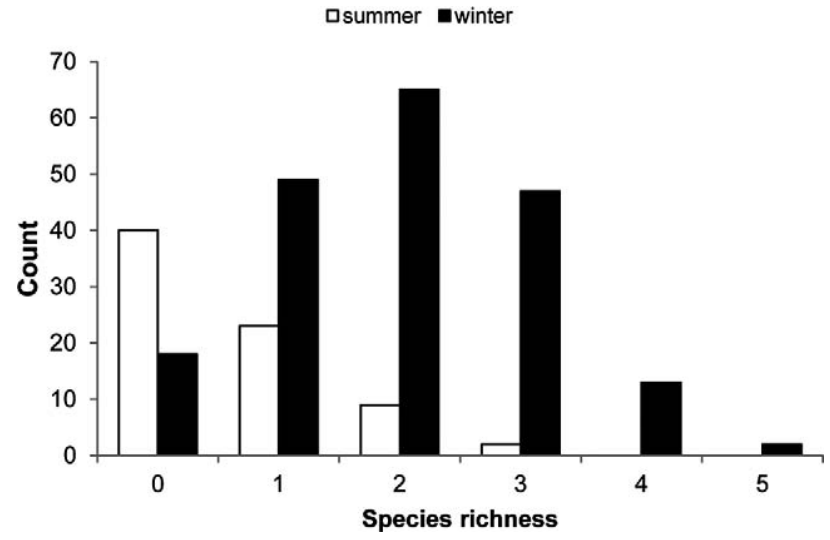

FIgure 3. Seasonal variation in ectoparasite species richness of Cryptomys hottentotus hottentotus sampled in the Northern and Western Cape Province of South Africa between February 2011 and August 2012.

grooming behavior (Dick and Patterson, 2008; Krasnov, 2008; Pap et al., 2013). Although the balanced sex ratio in E. hilli could be linked to low mobility of lice and the high dependence of both sexes on the host for nutrition, the pronounced male bias in the remaining species runs contrary to the common female bias in ectoparasite populations. This could partially be attributed to body size dimorphism as the larger sex (usually females) may be more costly to produce (Marshall, 1981; Pap et al., 2013); and indeed in all 3 species with male sex bias, females are larger than males (Till, 1963; Segerman, 1995; Baker, 1999). Alternatively, the unusual sex ratios observed in the current study might be linked to the host biology. Because common mole-rats live in groups that share a common nest (Bennett and Faulkes, 2000) and not all members of a group could be sampled, the ectoparasite sex ratio found on the entire host group or in the burrow system might differ from that observed on the animals sampled.

Clear seasonal patterns were apparent in both the prevalence and abundance of all ectoparasite species found in this study, with burdens being higher during the cool, wet winter. As a result, the species richness also varied with season. This mirrors the ectoparasite population dynamic of other rodent hosts occurring in the region (Matthee et al., 2007). In contrast, in the highveld mole-rat, ectoparasite burdens were greatest during the wet summer (Viljoen et al., 2011), suggesting that changes in humidity rather than temperature are responsible for the observed pattern. In fact, the importance of rainfall for ectoparasites have been repeatedly shown for various taxa (Vinarski et al., 2007; Krasnov et al., 2008; Korallo-Vinarskaya et al., 2009). The winter rainfall coincides with the reproductive activity of the common mole-rat (Spinks et al., 1999); hence, the seasonal patterns observed may also be a result of a synchronized reproductive cycle between ectoparasite species and their hosts (Rothschild and Ford, 1964; Christe et al., 2000; Lourenço and Palmeirim, 2008). Alternatively, but not mutually exclusive, host behavior could contribute to the observed seasonal dynamic of ectoparasite burdens because dispersal activity is strongly linked to rainfall (Spinks et al., 2000), and the resulting increased exchange of individuals between host groups may promote parasite transmission.

In conclusion, the ectoparasite species richness of the common mole-rat was low, with only 6 species, 3 of which were mites. The 
Androlaelaps spp. found are possibly specific to the Bathyergidae, whereas the flea and the louse species appear specific to $C . h$. hottentotus. All ectoparasite species were aggregated among hosts and sex biases were apparent in 4 of the 5 species. This high degree of host specificity could be linked to the subterranean niche exploited by the host. Both prevalence and abundance of ectoparasite species were greater during the winter rainfall,; however, the contributions of climate (i.e., rainfall pattern) and host behavior (i.e., dispersal pattern) cannot unambiguously be discerned.

\section{ACKNOWLEDGMENTS}

We thank the owners for access to their properties for animal capture and Northern and Western Cape Nature Conservation for issuing the capture permits. This research was approved by the Animal Ethics Committee of the University of Pretoria (EC005-11). We are indebted to many volunteers during fieldwork and to Eddie Green for help with louse identification. This work was funded by the NRF-SAR Chair for Mammalian Behavioural Ecology and Physiology to N.C.B. and a University of Pretoria Research Fellowship to H.L.

\section{LITERATURE CITED}

Altizer, S., A. Dobson, P. Hosseini, P. Hudson, M. Pascual, and P. RoHANI. 2006. Seasonality and the dynamics of infectious diseases. Ecology Letters 9: 467-484.

BAKER, A. S. 1999. Mites and ticks of domestic animals: An identification guide and information source. The Natural History Museum, London, U.K., 240 p.

Bennett, N. C., And C. G. FaulKes. 2000. African mole-rats: Ecology and eusociality. Cambridge University Press, Cambridge, U.K., 291 p.

Bush, A. O., K. D. Lafferty, J. M. Lotz, and A. W. Shostak. 1997. Parasitology meets ecology on its own terms: Margolis et al. revisited. Journal of Parasitology 83: 575-583.

Christe, P., R. Arlettaz, and P. Vogel. 2000. Variation in intensity of a parasitic mite (spinturnix myoti) in relation to the reproductive cycle and immunocompetence of its bat host (Myotis myotis). Ecology Letters 3: 207-212.

De GraAfF, G. 1964. On the parasites associated with the Bathyergidae. Koedoe 7: 113-123.

- 1972. On the mole-rat (Cryptomys damarensis) (Rodentia) in the Kalahari Gemsbok National Park. Koedoe 15: 25-35.

. 1981. The rodents of southern Africa, Butterworths, Durban. South Africa, $267 \mathrm{p}$.

Dick, C. W., And B. D. Patterson. 2008. An excess of males: Skewed sex ratios in bat flies (Diptera: Streblidae). Evolutionary Ecology 22: 757-769.

Durden, L. A. 1991. New records of sucking lice (Insecta: Anoplura) from African mammals. Journal of African Zoology 105: 331-342.

FABIYI, J. P. 1996. Association between duration of humid season and geographical distribution patterns of different species of chewing lice (Mallophaga: Insecta) infesting domestic chickens in Nigeria. Journal of Parasitology 82: 1034-1036.

Fain, A., F. S. Lukoschus, and M. Nadchatram. 1980. Malaysian parasitic mites II. Myobiidae (Prostigmata) from rodents. International Journal of Acarology 6: 109-120.

Faulkes, C. G., N. C. Bennett, M. W. Bruford, H. P. O’Brien, G. H. Aguilar, and J. U. M. Jarvis. 1997. Ecological constraints drive social evolution in the African mole-rats. Proceedings of the Royal Society B 264: 1619-1627.

- E. Verheyen, W. Verheyen, J. U. M. Jarvis, and N. C. Bennett. 2004. Phylogeographical patterns of genetic divergence and speciation in African mole-rats (Family: Bathyergidae). Molecular Ecology 13: 613-629.

Hamilton, W. D. 1967. Extraordinary sex ratios. Science 156: 477-488.

Henry, L.G., ANd S. McKeever. 1971. A modification of the washing technique for quantitative evaluation of the ectoparasite load for small mammals. Journal of Medical Entomology 8: 504-505.

Hickman, G. C. 1979. A live trap and trapping technique for fossorial mammals. South African Journal of Zoology 14: 9-12.
Ingram, C. M., H. Burda, And R. L. Honeycutt, 2004. Molecular phylogenetics and taxonomy of the African mole-rats, genus Cryptomys and the new genus Coetomys Gray, 1864. Molecular Phylogenetics and Evolution 31: 997-1014.

KLEIN, S. L. 2004. Hormonal and immunological mechanisms mediating sex differences in parasite infection. Parasite Immunology 26: 24722464.

Korallo-Vinarskaya, N. P., B. R. Krasnov, M. V. Vinarski, G. I. Shenbrot, D. Mouillot, and R. Poulin. 2009. Stability in abundance and niche breadth of gamasid mites across environmental conditions, parasite identity and host pools. Evolutionary Ecology 23: $329-345$.

Krasnov, B. R. 2008. Functional and evolutionary ecology of fleas. Cambridge University Press. Cambridge, U.K., 593 p.

, N. P. Korallo-Vinarskaya, M. V. Vinarski, G. I. Shenbrot, D. Mouillot, AND R. Poulin. 2008. Searching for general patterns in parasite ecology: Host identity versus environmental influence on gamasid mite assemblages in small mammals. Parasitology 135: 229242.

LEDGER, J. A. 1980. The arthropod parasites of vertebrates in Africa south of the Sahara. Volume IV. Phthiraptera (Insecta). South African Institute for Medical Research, Johannesburg, South Africa, $327 \mathrm{p}$.

Lourenço, S., and J. M. Palmeirim. 2008. Which factors regulate the reproduction of ectoparasites of temperate-zone cave-dwelling bats? Parasitology Research 104: 127-134.

Marshall, A. G. 1981. The sex ratio in ectoparasitic insects. Ecological Entomology 6: 155-174.

Martin, L. B., Z. M. Weil, and R. J. Nelson. 2008. Seasonal changes in vertebrate immune activity: mediation by physiological trade-offs. Philosophical Transactions of the Royal Society 363: 321-339.

Matthee, S., I. G. Horak, J.-C. Beaucournu, L. A. Durden, E. A. Ueckermann, and M. A. Mcgeoch. 2007. Epifaunistic arthropod parasites of the four-striped mouse, Rhabdomys Pumilio, in the Western Cape Province, South Africa. Journal of Parasitology 93: $47-59$.

Moore, S. L., AND K. WiLson. 2002. Parasites as a viability cost of sexual selection in natural populations of mammals. Science 297: 2015-2018.

Pap, P. L., A. Costica, I. V. Csongor, B. Zoltan, and V. Orsolya. 2013. Sex ratio and sexual dimorphism of three lice species with contrasting prevalence parasitizing the house sparrow. Journal of Parasitology 99: 24-30.

Poulin, R. 1993. The disparity between observed and uniform distributions: A new look at parasite aggregation. International Journal for Parasitology 23: 937-944.

. 2007. Evolutionary ecology of parasites. Princeton University Press, Princeton, New Jersey, $342 \mathrm{p}$.

Rossin, M. A., A. I. Malizia, J. T. Timi, and R. Poulin. 2010. Parasitism underground: determinants of helminth infections in two species of subterranean rodents Octodontidae). Parasitology 137: 1569-1575.

Rósza, L., J. Reiczigel, and G. Majoros. 2000. Quantifying parasites in samples of hosts. Journal of Parasitology 86: 228-232.

Rothschild, M., AND B. Ford. 1964. Breeding of rabbit flea (Spilopsyllus cuniculi (Dale)) controlled by the reproductive hormones of the host. Nature 201: 103-104.

Schalk, G., AND M. R. Forbes. 1997. Male biases in parasitism of mammals: Effects of study type, host age, and parasite taxon. Oikos 78: $67-74$.

Scharff, A., H. Burda, F. Tenora, M. Kawalika, and V. Barus. 1997. Parasites in social subterranean Zambian mole-rats (Cryptomys ssp., Bathyergidae, Rodentia). Journal of Zoology 241: 571-577.

Segerman, J. 1995. Siphonatera of Southern Africa: A Handbook for the identification of fleas. South African Institute for Medical Research. Johannesburg. South Africa, $264 \mathrm{p}$.

Soliman, S., A. S. Marzouk, A. J. Main, and A. A. Montasser. 2001. Effect of sex, size, and age of commensal rat hosts on the infestation parameters of their ectoparasites in a rural area of Egypt. Journal of Parasitology 87: 1308-1316.

Spinks, A. C., N. C. Bennett, and J. U. M. Jarvis. 1999. Regulation of reproduction in female common mole-rats (Cryptomys hottentotus hottentotus): The effects of breeding season and reproductive status. Journal of Zoology 248: 161-168.

- — AND . 2000. A comparison of the ecology of two populations of the common mole-rat, Cryptomys hottentotus hotten- 
totus: The effect of aridity on food, foraging and body mass. Oecologia 125: 341-349.

Tenora, F., V. Barus, M. Prokes, R. Sumbera, and B. Koubková. 2003. Helminths parasitizing the silvery mole-rat, Heliophobius argenteocinereus (Rodentia: Bathyergidae) from Malawi. Helminthologia 40: 153-160.

Till, W. 1963. Ethiopian mites of the genus Androlaelaps Berlese s. lat (Acari: Mesostigmata). Bulletin of The British Museum of Natural History 10: 1-104.

Van Daele, P. A. A., E. Verheyen, M. Brunain, and D. Adrieaens. 2007. Cytochrome b sequence analysis reveals differential molecular evolution in African mole-rats of the chromosomally hyperdiverse genus Fukomys (Bathyergidae, Rodentia) from the Zambezian region. Molecular Phylogenetics and Evolution 45: 142-157.

Viljoen, H., N. C. Bennett, E. A. Ueckermann, and H. Lutermann. 2011. The role of host traits, season and group size on parasite burdens in a cooperative mammal. PloS ONE 6: e27003.
Vinarski, M. V., N. P. Korallo, B. R. Krasnov, G. I. Shenbrot, and R. Poulin. 2007. Decay of similarity of gamasid mite assemblages parasitic on palaearctic small mammals: Geographic distance, hostspecies composition or environment. Journal of Biogeography 34: $1691-1700$.

Wilson, K., O. N. Burnstad, A. P. Dobson, S. Merler, G. Poglayen, S. E. Randolph, A. F. Read, and A. Skorping. 2001. Heterogeneities in macroparasite infections: Patterns and processes. In The ecology of wildlife diseases, P. J. Hudson, A. Rizzoli, B. T. Grenfell, H. Heesterbeek, and A. P. Dobson (eds.). Oxford University Press, New York, New York, p. 6-44.

ZuMPT, F. 1961. The arthropod parasites of vertebrates in Africa south of the Sahara. Vol. I (Chelicerata). South African Institute of Medical Research, Johannesburg, South Africa, 457 p.

1966. The arthropod parasites of vertebrates in Africa south of the Sahara. Vol. III (Insecta excl. Phthiraptera). South African Institute of Medical Research, Johannesburg, South Africa, 283 p. 